\title{
PREPARAÇÃO DE NANOPARTICULAS A PARTIR DA POLPA DE CELULOSE E DA CELULOSE MICROCRISTALINA UTILIZANDO LÍQUIDO IÔNICO
}

Suellen Rocha Vieira1; Jânia Betânia Alves da Silva ${ }^{1,2}$; Ussemane Massagy², Jorge Fernando Brandão; Valéria Carvalho Ebinuma ${ }^{2}$; Janice Izabel Druzian"; Carolina Oliveira de Souza ${ }^{1}$

${ }^{1}$ Programa de Pós-Graduação em Ciência de Alimentos; Faculdade de Farmácia; Universidade Federal da Bahia; Ondina-Salvador/Bahia, Brasil; e-mail: suelleneng.alimentos@hotmail.com

2 Departamento de Bioprocessos e Biotecnologia; Faculdade de Ciências Farmacêuticas; Universidade Estadual Paulista; Rodovia Araraquara-Jaú/São Paulo, Brasil.

Resumo: Este estudo teve como objetivo a preparação e caracterização de nanopartículas de celulose utilizando líquido iônico. As nanoparticulas de celulose foram preparadas a partir da polpa de celulose (PC) e celulose microcristalina (CM), utilizando o líquido iônico (LI) Hexalato de propilamônio (PA), com tempo de hidrolise de 120; 150 e 180 min, como controle utilizou-se o processo convencional com ácido sulfúrico $\left(\mathrm{H}_{2} \mathrm{SO}_{4}\right)$. As nanocelulose produzidas por LI-PA com CM apresentou maior rendimento. Por espectrômetro de transformada de Fourier (FTIR), foi possível indicar a obtenção de nanoparticulas de celulose com LI, os espectros obtidos das nanoparticulas de celulose foram similares entre si e com o controle. De acordo com os resultados obtidos, o líquido iônico é uma alternativa para substituição do método convencional.

Palavras-Chave: celulose; hexalato de propilamônio; hidrólise ácida e nanoparticulas.

\section{PREPARATION OF NANOPARTICULES FROM CELLULOSE PULP AND MICRO CRYSTALLINE CELLULOSE USING IONIC LIQUID}

\begin{abstract}
This study aimed to prepare and characterize cellulose nanoparticles using ionic liquid. The cellulose nanoparticles were prepared from cellulose pulp (PC) and microcrystalline cellulose $(\mathrm{CM})$ using ionic liquid (LI) propylammonium hexalate (PA), with hydrolysis time of $120 ; 150$ and $180 \mathrm{~min}$, as a control, the conventional sulfuric acid $\left(\mathrm{H}_{2} \mathrm{SO}_{4}\right)$ process was used. The nanocellulose produced by LI-PA with CM presented higher yield. By Fourier transform spectrometer (FTIR), it was possible to indicate the obtaining of cellulose nanoparticles with $\mathrm{LI}$, the spectra obtained from cellulose nanoparticles were similar to each other and to the control. According to the results obtained, the ionic liquid is an alternative to replace the conventional method.
\end{abstract}

Keywords: cellulose; propylammonium hexalate; acid hydrolysis and nanoparticles. 


\section{INTRODUÇÃO}

A celulose é o polímero biodegradável mais abundante existente na terra [1,2]. A sua hidrólise forma derivados como as nanocelulose, que tem sido utilizada em diferentes campos de aplicação como, nas indústrias de papel e tecidos, aplicações tecnológicas, embalagens de alimentos entre outros [3]. As nanocelulose consiste em propriedades de alta força, rigidez, resistência mecânica, alta propriedade térmica e maior área superficial [4].

A maioria dos métodos para produção de nanocelulose requerem altas temperaturas, altas pressões e concentrações. O principal método utilizado para sua produção é a hidrólise ácida, que hidrolisa as regiões amorfas da estrutura da celulose. O ácido mais utilizado nesse processo é o ácido sulfúrico [5]. A desvantagem desse método tradicional para a produção é devido aos danos ambientais causados [2]. Embora o tratamento biológico com enzimas possa ser realizado sob condições de reações suaves, essa abordagem é muito lenta e principalmente a baixa solubilidade da lignina [6]. Um solvente ideal para dissolver a celulose deve atender aos critérios de alta solubilidade, rápida dissolução e baixa toxicidade [7].

Para melhorar a segurança e a compatibilidade com o meio ambiente na produção de nanocelulose, os líquidos iônicos (LIs) vêm sendo utilizados como substitutos ideais da hidrólise ácida [8]. Os LIs são um grupo de sais com ponto de fusão menor que $100^{\circ} \mathrm{C}$, capazes de dissolver a celulose, quebrando as ligações de hidrogênio $[9,10]$.

Atualmente surge um grande interesse na utilização dos Lls para a produção de nanocelulose, devido à possibilidade de produzi-las com um solvente que não apresenta riscos ambientais, além de apresentar alta estabilidade térmica, estabilidade química, não inflamável, baixa pressão de vapor, baixo ponto de fusão, miscibilidade e recuperação $[3,5,9,10]$.

Entre os novos potenciais solventes, os líquidos iônicos foram considerados 0 "Alkahest" nas últimas duas décadas, já que podem ser utilizados para a dissolução de polissacarídeos nativos e proteínas [7]. No entanto, um processo verde, econômico e eficiente para a hidrólise da celulose sob condições de reação moderadas, apresentam poucos estudos. Assim, este trabalho teve como objetivo a preparação e caracterização de nanoparticulas de celulose utilizando o líquido iônico da família imidazolium.

\section{METODOLOGIA}

\subsection{Material}

A Polpa de celulose comercial de eucalipto foi doada pela Indústria Bahia pulpBahia, Brasil. Celulose microcristalina foi adquirida pela Synth. O Líquido lônico Hexalato de Propilamônio (LI-PA), foi sintetizado e doado pelo grupo de pesquisa Bioppul (Araraquara-SP/Brasil). 


\subsection{Preparação das Nanoparticulas de Celulose}

As nanocelulose foram preparadas utilizando dois tratamentos. Inicialmente foi realizada a hidrólise da polpa de celulose (Controle) em solução de ácido sulfúrico $\left(\mathrm{H}_{2} \mathrm{SO}_{4}\right)$ a $45 \%$ na proporção de $1: 9(\mathrm{~m} / \mathrm{m})$ por $180 \mathrm{~min}$ a $80^{\circ} \mathrm{C}$. A preparação das nanoparticulas de celulose com o LI-PA, foi realizada na proporção de 1:9 de polpa de celulose (PC) e celulose microcristalina (CM) em LI-PA. As amostras foram agitadas (Agitador Digital IKA® RW20) a $400 \mathrm{rpm}$ e aquecida (IKA C-MAG HS7) a $80 \pm 2^{\circ} \mathrm{C}$. Alíquotas foram retiradas em três tempos diferentes $(120 ; 150$ e $180 \mathrm{~min})$, e transferidas para tubos Falcon de $50 \mathrm{~mL}$. As suspensões foram centrifugadas (EppendorfCentrifuge S702R) a $7.000 \mathrm{rpm}$ por $30 \mathrm{~min}$, os três primeiros sobrenadantes foram coletados e armazenados para recuperação dos líquidos por evaporação. As amostras foram então lavadas com água destilada e centrifugadas a 7.000 rpm por 10 min, para obtenção das nanopartículas.

\subsection{Caracterização das Nanoparticulas de Celulose}

O cálculo do rendimento foi determinada por teste de secagem em estufa de acordo, com a metodología proposta por Celestino (2010). Os espectros de FTIR foram obtidos na faixa de 4.000 a $400 \mathrm{~cm}^{-1}$ usando um Analisador IRPrestige -21, a partir de nanoparticulas de celulose dispersa em $\mathrm{KBr}$.

\section{RESULTADOS E DISCUSSÃO}

Os resultados expressos na Tabela 1 mostram que o rendimento da preparação das nanoparticulas de celulose a partir do líquido iônico (LI-PA), foi menor que o obtido com ácido (controle). A polpa de celulose (PC) no tempo de $180 \mathrm{~min}$, apresentou o maior rendimento, com valor de $0,171 \mathrm{~g} / \mathrm{L}$, correspondente a $25,1 \%$ em relação ao controle, seguido por $150 \mathrm{~min}(0,126)$ e $120 \mathrm{~min}(0,101)$. Indicando que quanto maior o tempo de hidrolise maior produção das nanoparticulas, inferindo que o LI-PA precisa de maior tempo para hidrolisar as regiões amorfas da polpa de celulose.

Tabela 1. Rendimento das nanoparticulas de celulose obtidas com Líquido lônico e ácido sulfúrico (controle)

\begin{tabular}{|c|c|c|c|c|}
\hline Tratamentos & Teste & Tempo $(\mathrm{min})$ & $\mathrm{g} / \mathrm{L}$ & $\mathrm{g} / \mathrm{g}$ Polpa \\
\hline Controle & Polpa de Celulose & 180 & 0,680 & 0,0102 \\
\hline \multirow{4}{*}{ LI-PA } & Polpa de Celulose - & 120 & 0,101 & 0,0010 \\
\cline { 3 - 5 } & PC & 150 & 0,126 & 0,0012 \\
\cline { 2 - 4 } & & 180 & 0,171 & 0,0017 \\
\cline { 2 - 4 } & \multirow{2}{*}{\begin{tabular}{c} 
Celulose \\
\cline { 2 - 4 }
\end{tabular}} & 120 & 0,226 & 0,0022 \\
\cline { 2 - 4 } & Microcristalina - CM & 150 & 0,226 & 0,0022 \\
\cline { 2 - 4 } & & 180 & 0,072 & 0,0007 \\
\hline
\end{tabular}


De acordo com os resultados, o tempo de hidrólise foi diretamente proporcional ao aumento do rendimento das nanoparticulas de celulose obtidas pelo tratamento $\mathrm{LI}$ PA com a polpa de celulose de eucalipto. O mesmo comportamento não foi observado quando utilizado a celulose microcristalina, onde o maior rendimento foi encontrado em 120 e $150 \mathrm{~min}(\sim 0,226 \mathrm{~g} / \mathrm{L})$, seguido por $180 \mathrm{~min}(0,072 \mathrm{~g} / \mathrm{L})$, indicando que o LIPA degrada mais rapidamente a celulose microcristalina do que a polpa de celulose. No entanto, para maiores conclusões, são necessárias análises mais específicas (Difração por Raio X - DRX e Microscopia Eletrônica de Transmissão-MET) para avaliar a estrutura e a morfologia das nanoparticulas de celulose produzidas. Mao et al., (2013), preparou nanopartículas de celulose com o LI 1-butil-3-metilimidazólio sulfato $\left([\mathrm{Bmim}] \mathrm{HSO}_{4}\right)$, utilizando celulose microcristalina e encontrou um rendimento de $48 \pm 2 \%$ com o processo de hidrólise a $120^{\circ} \mathrm{C}$ por $24 \mathrm{~h}$.

Nas Figuras 1 e 2 estão os espectros de infravermelho das nanoparticulas de celulose preparadas com LI-PA. Os espectros obtidos das amostras foram similares entre si tanto a PC quanto a MC, apresentando apenas diferença na intensidade das bandas.

Figura 1. Espectro de FTIR das nanocelulose obtidas por hidrólise com ácido sulfúrico (controle) e Líquido lônico (LI-PA) com Polpa de Celulose (PC)

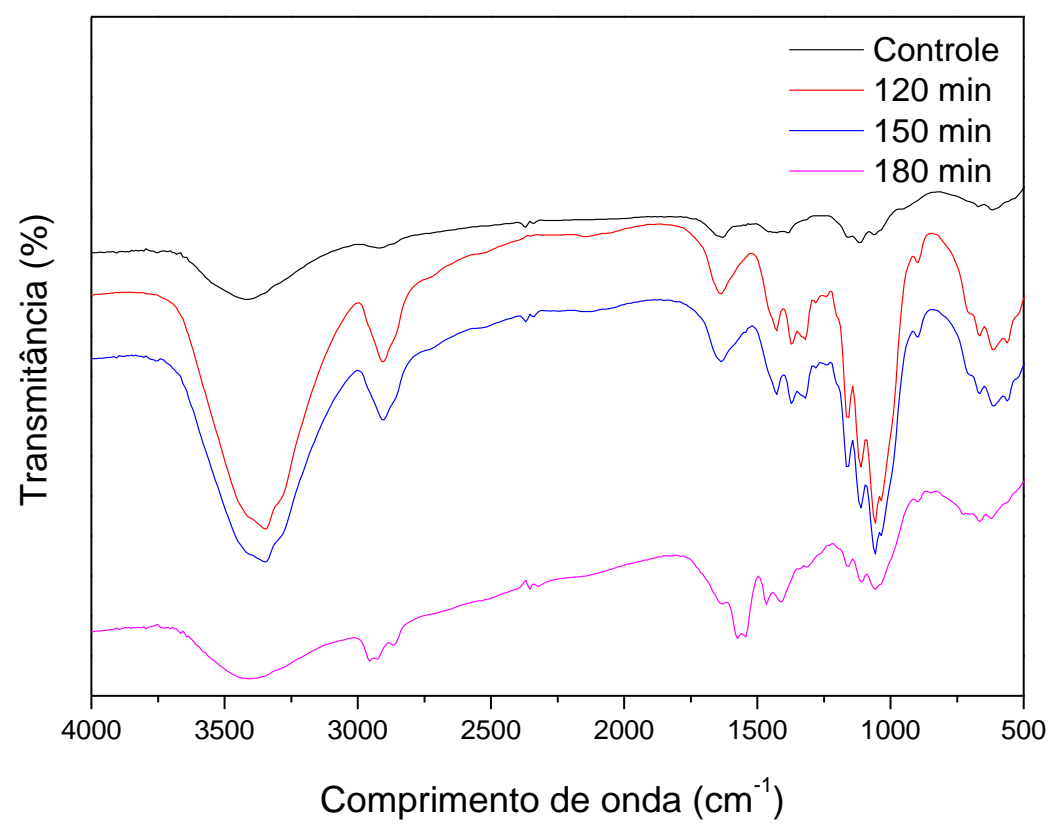

As bandas de absorção na região de $3300 \mathrm{~cm}^{-1}$ são referentes a $\mathrm{O}-\mathrm{H}$ associado. As bandas na faixa de $2900 \mathrm{~cm}^{-1} \mathrm{e} 1640 \mathrm{~cm}^{-1}$ são referentes aos grupos $\mathrm{CH} 2$. As deformações angulares simétricas de grupos metileno são observados na região de $1435 \mathrm{~cm}^{-1}$ [3]. Bandas na faixa de $1370 \mathrm{~cm}^{-1}$ e $1050 \mathrm{~cm}^{-1}$ são vibrações de flexão dos anéis aromáticos dos polissacarídeos e anéis presentes na estrutura da celulose [13]. As bandas em $893 \mathrm{~cm}^{-1}$ são características das ligações glicosídicas [14], na região de 
$610 \mathrm{~cm}^{-1}$ são deformações, oscilações e torção [5,15]. Os espectros mostram que o processo de hidrólise com LI-PA nos dois testes (PC e CM) são eficientes, devido à similaridade da estrutura química entre eles e o controle.

Resultados similares foram encontrados por Gonçalves et al., (2018), para as nanocelulose, onde bandas de $\mathrm{O}-\mathrm{H}$ foram observadas no espectro na faixa de 3387 a $2899 \mathrm{~cm}^{-1}$, como também bandas na região de $1646 \mathrm{~cm}^{-1} ; 1425 \mathrm{~cm}^{-1}$ e $1056 \mathrm{~cm}^{-1}$. As bandas na faixa de $1640 \mathrm{~cm}^{-1}$ e $1430 \mathrm{~cm}^{-1}$ também foram encontradas por Man et al., (2011).

Figura 2. Espectro de FTIR das nanocelulose obtidas por hidrólise com ácido sulfúrico (controle) e Líquido lônico (LI-PA) com Celulose Microcristalina (MC)

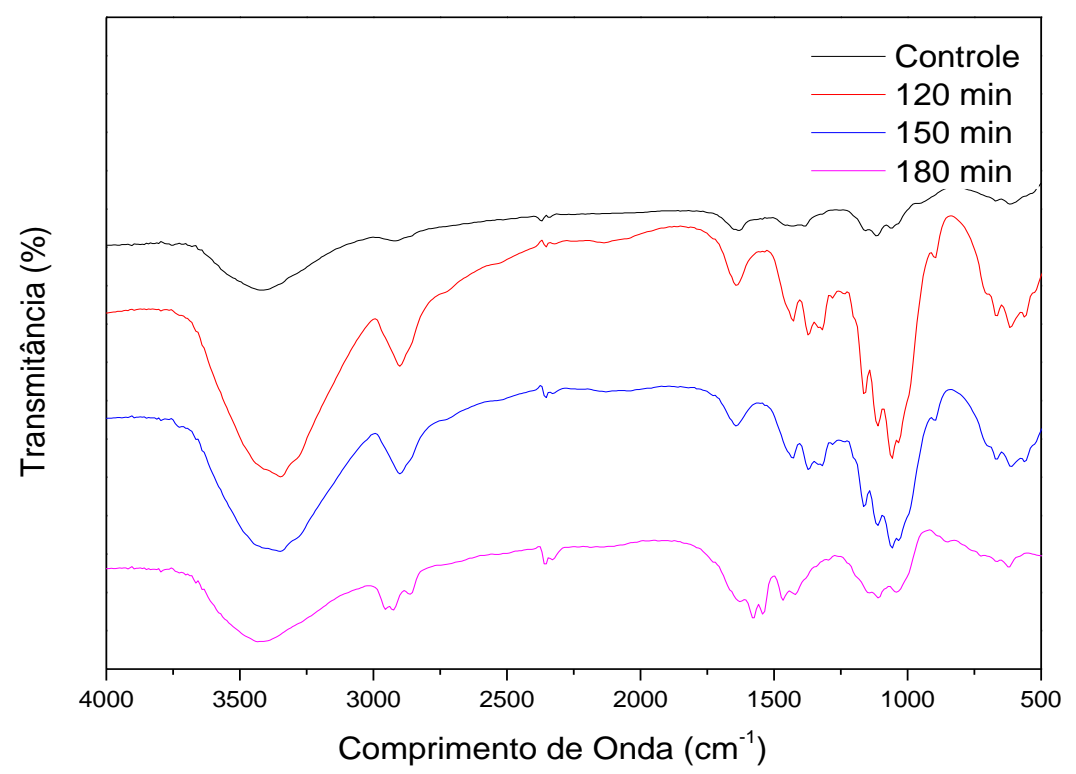

\section{CONCLUSÃO}

O presente estudo mostrou que o líquido iônico PA foi eficiente na preparação das nanoparticulas de celulose comparado com o processo de hidrólise convencional $\left(\mathrm{H}_{2} \mathrm{SO}_{4}\right)$. Como foi verificado nos espectros da análise de FTIR, as nanoparticulas, apresentaram mesmas bandas presentes no espectro da nanoparticula obtida pelo método convencional. No entanto, são necessários estudos adicionais para caracterizar as nanoparticulas de celulose obtidas a partir da hidrólise com Líquido lônico. 
Agradecimentos

Agradecimento a Fapesb pelo financiamento do projeto ( $\mathrm{n}^{\circ}$ BOL0684/2019), a Indústria Bahiapulp-Bahia e o grupo de pesquisa Bioppul pelas doações.

\section{REFERÊNCIAS}

${ }^{1}$ SWATLOSKI, R. P.; SPEAR, S. K.; HOLBREY, J. D.; ROGERS, R. D. Dissolução de Celulosecom Líquidos lônicos.Journal of the American ChemicalSociety, v. 124, n. 18, p. 4974-4975, 2002.

2 MEENATCHI, B.; RENUGA, V.; MANIKANDAN, A. Cellulose dissolution and regeneration using various imidazolium based protic ionic liquids. Journal of Molecular Liquids, v. 238, p. 582-588, 2017.

${ }^{3}$ GONÇALVES, A. P.; OlIVEIRA, E.; MATTEDI, S.; JOSÉ, N. M. Separation of cellulose nanowhiskers from microcrystalline cellulose with an aqueous protic ionic liquid based on ammonium and hydrogensulphate. SeparationandPurification Technology, v. 196, p. 200-207, 2018.

4 PHANTHONG, P.; REUBROYCHAROEN, P.; HAO, X.; XU, G.; ABUDULA, A.; GUAN, G. Nanocellulose: Extraction and application. Carbon Resources Conversion, v. 1, n. 1, p. 32-43, 2018.

${ }^{5}$ MAN, Z.; MUHAMMAD, N.; SARWONO, A.; BUSTAM, M. A.; KUMAR, M. V.; RAFIQ, $S$. Preparation of cellulose nanocrystals using an ionic liquid. Journal Polymers and the Environmen, v. 19, p. 726-731, 2011.

${ }^{6}$ MONIRUZZAMAN, M.; ONO, T. Separation and characterization of cellulose fibers from cypress wood treated with ionic liquid prior to laccase treatment. Bioresource Technology, n. 127, p. 132-137, 2013.

7 XU, H.; BRONNER, T.; YAMAMOTO, M.; YAMANE, H. Regeneration of cellulose dissolved in ionic liquid using laser-heated meltelectrospinning. Carbohydrate Polymers, v. 201, p.182-188, 2018.

8 ZHAO, H.; XIA, S.; MA, P. Review Use of ionic liquids as 'green' solvents for extraction. Journal of Chemical Technology \& Biotechnology, v. 80, p.1089-1096, 2005.

9 VRANES, M.; PANIC, J.; TOT, A.; OSTOJIC, S.; CETOJEVIC-SIMIN, D.; JANKOVIC, N.; GADZURIC, S. Synthesis and thermophysical characterization of new biologically friendly agmatine-based ionic liquids and salts by experimental and computational approach. ACS Sustainable Chemistry \& Engineering, v. 7, p. 10773-10783, 2019.

10 LÓPEZ, F. I.; DOMÍNGUEZ, J. M.; MIRANDA, A. D.; TREJO-DURÁN, M.; ALVARADO-MÉNDEZ, E.; VÁZQUEZ, M. A. Synthesis of symmetric ionic liquids and their evaluation of nonlinear optical properties. Optical Materials, v. 96, p. 109276, 2019. 
11 CELESTINO, S.M.C. "Documentos / Embrapa Cerrados: Princípios de Secagem de Alimentos". Documentos 276, Planaltina, p. 51, jan 2010.

12 MAO, J.; OSORIO-MADRAZO, A.; LABORIE, M. P. Preparation of cellulose I nanowhiskers with a mildly acidic aqueous ionic liquid: reaction efficiency and whiskers attributes. Cellulose, n. 20, p. 1829-1840, 2013.

13 VESTENA, M.; GROSS, I. P.; MULLER, C. M. O.; PIRES, A. T. N. Isolamento de whiskers de fontes naturais e seus dispersos em meio não aquoso. Polímeros, v. 26, n. 4, p. 327-335, 2016.

${ }^{14}$ LAN, W.; LIU, C. F.; YUE, F. X.; SUN, R. C.; KENNEDY, J. F. Ultrasound-assisted dissolution of cellulose in ionic liquid. Carbohydrate Polymers, v. 86, p. 672-677, 2011. 15 HAAFIZ, M. K.; HASSAN, A.; ZAKARIA, Z.; INUWA, I. M. Isolation and characterization of cellulose nanowhiskers from oil palm biomass microcrystalline cellulose, Carbohydrate Polymers v.103, p.119-125, 2014. 